\title{
Investigación exploratoria sobre el efecto del aceite de motor usado en un suelo fino de subrasante
}

\section{Exploratory research on the effect of used engine oil on a subgrade fine soil}

\author{
R.D. Del Castillo ${ }^{(*)}, \underline{\text { A. Orobio }}^{(* * 2}$
}

\section{RESUMEN}

La búsqueda de nuevas alternativas para estabilizar suelos con desechos motivó la realización de esta investigación exploratoria para evaluar en forma preliminar las propiedades geotécnicas de un suelo fino de subrasante mezclado con diferentes porcentajes (4\%, 8\%, $12 \%$ y 16\%) de Aceite de Motor Usado (AMU). En el desarrollo de esta investigación se realizaron ensayos de límites de Atterberg, coeficiente de permeabilidad $(k)$, compresión inconfinada $\left(q_{u}\right)$, y relación humedad-densidad de un suelo clasificado como MH. Los ensayos se realizaron tanto para el suelo sin estabilizar como para el suelo estabilizado con AMU. Los resultados muestran que los limites líquido y plástico tienden a disminuir con el incremento de AMU, la densidad seca máxima se incrementa ligeramente, el contenido de humedad optima disminuye significativamente, la resistencia a la compresión inconfinada $\left(q_{u}\right)$ se mantiene constante para contenidos de AMU hasta el $8 \%$ y la permeabilidad $(k)$ no muestra variación significativa.

Palabras clave: Estabilización de suelos, subrasante, permeabilidad de suelos, aceite de motor usado.

\section{ABSTRACT}

The search for new alternatives for soil stabilization by means of wasted materials promoted the development of this exploratory research to perform a preliminary evaluation of the geotechnical properties of a fine subgrade soil stabilized with different percentage (4\%, 8\%, $12 \%$ y 16\%) of Used Engine Oil (UEO). In the development of this research, tests of Atterberg limits, permeability coefficient $(k)$, unconfined compression stress $\left(q_{u}\right)$, and water-density ratio were performed on a soil classified as MH. The tests were performed both on the unstabilized soil and on UEO stabilized soil. Results show that the liquid and plastic limits tend to lower as the percent of UEO increases, the maximum dry density lightly increase, the optimal water content lower significantly, the unconfined compression stress $\left(q_{u}\right)$ stays constant with up to $8 \%$ of UEO and permeability $(k)$ does not show significant variation.

Keywords: Soil stabilization, subgrad, soil permeability, used engine oil.

(*) Ingeniero Civil y Magíster en Ingeniería de la Universidad del Valle, Cali (Colombia) ruben.del@correounivalle.edu.co (R.D. Del Castillo).

(**) PhD. en Ing. Civil, Escuela de Ingeniería Civil y Geomática, Universidad del Valle, Cali (Colombia).

Persona de contacto/Corresponding author: armando.orobio@correounivalle.edu.co (A. Orobio).

ORCID: https://orcid.org/oooo-0002-5251-6389 (R.D. Del Castillo); https://orcid.org/oooo-0001-7166-3061 (A. Orobio).

Cómo citar este artículo/Citation: Del Castillo, R.D.; Orobio, A. (2020). Investigación exploratoria sobre el efecto del aceite de motor usado en un suelo fino de subrasante. Informes de la Construcción, 72(558): e336. https://doi.org/10.3989/ic.69016

Copyright: ( ) 2020 CSIC. Este es un artículo de acceso abierto distribuido bajo los términos de la licencia de uso y distribución Creative Commons Reconocimiento 4.0 Internacional (CC BY 4.0). 


\section{INTRODUCCIÓN}

Los efectos adversos en los pavimentos frecuentemente se atribuyen a la permeabilidad y contracto-expansión del suelo de la subrasante. Cuando el agua fluye a través de una masa de suelo, produce diferentes efectos negativos sobre esta, e.g., puede generar cambios en el peso volumétrico, degradación de los materiales, movimientos diferenciales en suelos expansivos, disminución del esfuerzo efectivo y de la resistencia al cortante debido a la presión del agua (1). Los drenajes juegan un papel fundamental en el desempeño de las estructuras de pavimento, no obstante, cuando el agua es capaz de filtrarse en subrasantes finas, se generan cambios en la humedad y variación en el volumen perjudicando a toda la estructura.

De esta manera, el agua influye en el comportamiento de las estructuras de pavimento, disminuyendo y afectando la resistencia de sus materiales, e incluso induciendo presiones hasta destruir la capa de rodadura. Para evitar este tipo de problema, existen diversas alternativas para estabilizar o mejorar las propiedades mecánicas de la subrasante, tales como las estabilizaciones con polímeros, cenizas volátiles, cal y productos residuales como la cáscara de arroz y la vinaza de la caña de azúcar.

Esta investigación busca de forma preliminar evaluar el comportamiento geotécnico de una subrasante mezclada con Aceite de Motor Usado (AMU), el cual en el medio colombiano es un residuo producido en grandes volúmenes y desafortunadamente en varias ocasiones termina en ríos y depósitos naturales, generando efectos nocivos para medio ambiente y la salud humana.

Como investigación exploratoria, los ensayos realizados permiten describir el comportamiento geotécnico del suelo en presencia de AMU, e incentiva a que futuras investigaciones enfoquen sus esfuerzos en otros aspectos geotécnicos, como las propiedades mecánicas o el efecto a largo plazo de distintos tipos de suelos en presencia de AMU.

En investigaciones previas de distintas partes del mundo, se ha utilizado aceite de motor de diferentes propiedades e incluso petróleo, para simular derrames de crudo y evaluar el comportamiento del suelo $(2,3,4,5,6,7,8,9,10)$. Sin embargo, hasta ahora no se ha experimentado el uso del AMU con fines geotécnicos.

Las investigaciones anteriores han encontrado que el coeficiente de permeabilidad de arcillas naturales decrece substancialmente con adiciones entre el o\% y $8 \%$ de aceite de motor 10W30 (10). Otros autores han encontrado una correlación inversa entre la permeabilidad y el contenido de aceite o petróleo crudo en suelos CL, SM y SP $(3,4,8)$.

Esta investigación exploratoria se enfoca en el análisis de las propiedades mecánicas del suelo mezclado con AMU, se requiere mayor investigación sobre los aspectos ambientales de estabilizar suelos con aceites, en especial la migración del aceite en el suelo y la contaminación del agua. La experiencia empírica de los autores indica que, cuando se estabilizan suelos finos cohesivos con bajos porcentajes de AMU, se logra una mezcla homogénea e impermeable, en la que aceite tiende a permanecer en el suelo estabilizado, con lo que se evita la contaminación del agua y los suelos, pero esto requiere posterior investigación.

\section{ACEITES LUBRICANTES}

Los aceites lubricantes son productos elaborados a partir de la destilación bajo vacío de la fracción de fueloil del petróleo crudo, adicionalmente están conformados por componentes aromáticos inestables y otros componentes removidos por extracción del solvente (11).

Estos aceites por efecto del uso pierden sus cualidades originales debido a que sufren cambios en sus propiedades físicas y químicas. Los cambios respecto a su composición inicial dependen de la presencia del agua, de la dilución del aceite por combustible, del hollín y metales producidos por el desgaste del motor. De igual manera, típicamente los aceites usados están conformados por mezclas de diferentes lubricantes, agua, hidrocarburos livianos, carbón, polímeros, fenoles, y metales como Pb, P, Ca y Zn (11). La Tabla 1 presenta las características típicas de los aceites usados en Colombia.

Tabla 1. Características de los aceites usados en Colombia (12).

\begin{tabular}{|l|c|c|}
\hline \multicolumn{1}{|c|}{ Características } & Automotor & Industrial \\
\hline Viscosidad a $40{ }^{\circ} \mathrm{C}, \mathrm{SSU}$ & $97-120$ & $143-330$ \\
\hline Gravedad $15.6{ }^{\circ} \mathrm{C},{ }^{\circ} \mathrm{API}$ & $19-22$ & $25.7-26.2$ \\
\hline Peso Específico a $15.6{ }^{\circ} \mathrm{C}$ & $0.9396-0.8692$ & $0.9002-0.8972$ \\
\hline Agua, \% Vol. & $0.2-33.8$ & $0.1-4.6$ \\
\hline Sedimentos, \% Vol. & $0.1-4.2$ & 0.0 \\
\hline Insolubles en Benceno, \% peso & $0.56-33 \cdot 3$ & 0.0 \\
\hline Solubles en gasolina, \% vol. & $2.0-9.7$ & 0.0 \\
\hline Punto de ignición, ${ }^{\circ} \mathrm{C}$ & $78-220$ & $157-179$ \\
\hline Poder Calorífico, $\mathrm{MJ} / \mathrm{kg}$ & $31.560-44.880$ & $40.120-41.840$ \\
\hline
\end{tabular}

Los aceites usados también suelen contener bifenoles policlorados, conocidos como PCB's, unos compuestos químicos con alta resistencia a la descomposición química, biológica y térmica. Estos compuestos son atractivos en la industria por ser considerados buenos conductores de calor y aislantes térmicos. Sin embargo, también son nocivos para la salud y el medio ambiente. Por fortuna, estudios y pruebas de laboratorios realizados en Colombia, concluyen que los aceites usados en circulación nacional no contienen presencia significativa de PCB's (12).

En Colombia se generan anualmente grandes volúmenes de aceites usados, provenientes del consumo de lubricantes por el sector automotor, de aceites de proceso y aceites de transformadores, entre otros (12). La Tabla 2 estima los volúmenes de aceites usados generados en algunas ciudades principales del país.

Tabla 2. Volumen de aceite usado generado y su distribución en el mercado (12).

\begin{tabular}{|l|l|c|}
\hline \multicolumn{1}{|c|}{ Zona } & \multicolumn{1}{c|}{ Ubicación } & Millones de Gal/Año \\
\hline Centro & Bogotá & 6.0 \\
\hline Norte & Barranquilla & 2.65 \\
\hline Sur & Cali & 2.93 \\
\hline Occidente & Medellín & 3.18 \\
\hline Total & & $\mathbf{1 4 . 7 6}$ \\
\hline
\end{tabular}

Infortunadamente, estudios del Ministerio de Minas y Energía indican que estos aceites se usan como combusti- 
bles en forma indiscriminada y sin tratamiento. La disposición de parte de estos volúmenes de aceites producidos en el país es diversa, aproximadamente 540 barriles por día son incorporados al mercado de los combustibles usados, como talleres de fundición, talleres de metalmecánica, textileras, siderúrgicas pequeñas, fábricas de conexiones, lavanderías, etc. (12).

Es preciso señalar el problema desde el ámbito ambiental, porque aunque en el medio colombiano existe el decreto 283 de 1960, donde se obliga a todos los sitios dedicados al cambio de aceite y filtros a destruir los envases y cambiar los filtros, de manera técnica y ambientalmente adecuada, sigue existiendo una grave disposición final en el medio ambiente, contaminando los ríos, afectando la permeabilidad en las tierras y generando dioxinas cancerígenas por su combustión (11). Una alternativa ambientalmente amigable, es utilizar el residuo del aceite usado en procesos de construcción, por lo que es importante investigar las propiedades ingenieriles de suelos estabilizados con residuo de aceite de motor y evaluar las alternativas de uso.

\section{METODOLOGIA}

\subsection{Selección del suelo y toma de muestras alteradas}

Los suelos cohesivos limos o arcillas son de amplia presencia mundial, además predominan como subrasantes de construcción en las vías de Colombia, particularmente en la ciudad de Santiago de Cali, motivo por el cual se eligió este tipo de suelos para el desarrollo de la investigación exploratoria.

Para seleccionar el suelo se consultaron los documentos técnicos disponibles como mapas, fotos y perforaciones previas en la ciudad Santiago de Cali y en algunos municipios cercanos. También se consultó el Informe No.4 del estudio de la microzonificación sísmica de Cali (MZSC) referente a "Investigaciones y zonificación geotécnica de la ciudad de Santiago de Cali", donde se subdivide la ciudad en zonas geotécnicamente homogéneas y se encuentra información como el tipo, la distribución de los materiales, las propiedades índices, la resistencia y la clasificación según el Sistema Unificado de Clasificación de Suelos (USCS) (13).

Según este informe, la mayoría de los estratos finos comienzan superficialmente entre o y $1 \mathrm{~m}$ hasta el rango 5 a $10 \mathrm{~m}$ en toda el área de estudio, exceptuando zonas como Pance y Navarro en el sur y oriente de la ciudad de Cali respectivamente. Por lo cual se decidió tomar la muestra de un terreno ubicado en el barrio Valle de Lili, de la comuna 17 del sur de la ciudad de Cali.

\subsection{Aceite de motor usado (AMU) y mezclas con el suelo}

Debido a que la investigación pretendía evaluar el efecto geotécnico de cualquier AMU en un suelo fino típico de subrasante, se obtuvieron aproximadamente 15 litros de este aceite usado en una estación de servicio del sur de la ciudad de Cali, donde eventualmente extraen este residuo del sector automotriz. Para cálculos de ensayos posteriores se determinó la gravedad específica del aceite usado por medio de la norma ASTM D1298 (14). Las características típicas de estos aceites se encuentran en la Tabla 1.

En función de determinar la variación de las propiedades del suelo en presencia de AMU, se realizaron límites de consistencia, ensayos modificados de compactación, resistencia a compresión inconfinada y finalmente se determinó el coeficiente de permeabilidad del suelo natural y del mismo con dosificaciones de $4 \%, 8 \%, 12 \%$ y $16 \%$ de aceite con base en peso seco del suelo. Para las mezclas, el suelo se secó al horno a $105^{\circ} \mathrm{C}$ para todos los ensayos, se tamizó por la malla de acuerdo al ensayo que se fuera a ejecutar, siempre siguiendo la normativa del Instituto Nacional de Vías (INVIAS) (15), se dosificó el aceite y se mezcló manualmente hasta obtener una mezcla homogénea de Suelo-AMU.

\subsection{Gravedad específica de sólidos}

Este valor es necesario para calcular la relación de vacíos de un suelo, se determinó por el método del picnómetro a través de la norma INV E - 128, la cual consistió en hallar el volumen equivalente de agua que ocupa una cantidad de partículas de sólidos que se encuentran en un matraz con agua. Conociendo este volumen y el peso de las partículas que han sido pesadas previamente, se determina el peso unitario de los sólidos.

\subsection{Curva granulométrica mediante USCS y límites de consistencia o límites de Atterberg}

Para realizar la curva granulométrica se realizó el análisis granulométrico por lavado y por medio del hidrómetro a través de las normas INV E - 123 e INV - 124 respectivamente. Para el análisis por tamizado se emplearon 300 gramos de suelo seco en el horno a $105^{\circ} \mathrm{C}$, y para la fracción más fina $(75 \mu \mathrm{m})$ se usaron 50 gramos de suelo en una solución de hexametafosfato de sodio al $4 \%$ durante un día.

Para la clasificación de un suelo fino mediante el USCS, además de que el porcentaje de finos que pasa la malla No.200 debe ser mayor al 50\%, es necesario determinar los límites de consistencia del suelo. Estos son los contenidos de agua para los cuales el suelo cambia de un estado físico a otro. Para encontrarlos se siguió la norma INV E - 125 e INV E - 126.

\subsection{Ensayo modificado de compactación}

Para la ejecución de los ensayos de compactación se siguió la norma INV E - 142 (ensayo de compactación modificado). El objetivo de este ensayo es determinar la relación entre la humedad y la masa unitaria de los suelos compactados en un molde de tamaño dado con un martillo de $4.54 \mathrm{~kg}$ que cae de una altura de $45.72 \mathrm{~cm}$. Para el suelo natural se siguió el método B y para la mezcla suelo el AMU se utilizó el método A con el propósito de optimizar los materiales y el proceso de mezclado. Ambos métodos tienen energía de compactación equivalente.

\subsection{Ensayo de compresión inconfinada}

El ensayo de compresión inconfinada permite determinar un valor aproximado de la resistencia de los suelos cohesivos en términos de esfuerzos totales, bajo condiciones inalteradas o 
remoldeadas. De acuerdo a la resistencia obtenida, es posible calificar la consistencia del suelo de acuerdo a la Tabla 3.

Tabla 3. Consistencia del suelo según su resistencia a la compresión inconfinada (16).

\begin{tabular}{|l|c|c|}
\hline \multirow{2}{*}{$\begin{array}{c}\text { Consistencia } \\
\text { del suelo }\end{array}$} & Resistencia a la Compresión Inconfinada \\
\cline { 2 - 3 } & $\mathbf{k g f} / \mathbf{c m}^{2}$ & $\mathbf{( k P a )}$ \\
\hline Muy blanda & $<0.25$ & $(<25)$ \\
\hline Blanda & $0.25-0.50$ & $(25-50)$ \\
\hline Mediana & $0.5-1.00$ & $(50-100)$ \\
\hline Firme & $1.00-2.00$ & $(100-200)$ \\
\hline Muy firme & $2.00-4.00$ & $(200-400)$ \\
\hline Dura & $>4.00$ & $(>400)$ \\
\hline
\end{tabular}

Para realizar el ensayo se adoptó la norma INV E - 152. Donde se especifica que las muestras a ensayar deben tener sección constante y eje perpendicular a dicha sección. El diámetro debe ser mayor a $30 \mathrm{~mm}$, la partícula máxima contenida debe ser menor o igual a 1/10 del diámetro y la relación de la altura al diámetro se debe encontrar entre 2 a 2.5.

Para cumplir estas especificaciones y con el fin de evaluar la influencia del AMU en la resistencia a compresión inconfinada del suelo, se decidió ensayar cada muestra, remoldeándola a la densidad seca máxima y contenido óptimo de humedad obtenidos en los ensayos modificados de compactación. Las muestras se armaron en un cilindro metálico hueco de volumen previamente determinado, compactando el material con un disco metálico que se desliza al interior del cilindro. Posteriormente el espécimen compacto se extraía del molde para ser ensayado; se prepararon dos para cada contenido de aceite empleado, incluyendo el o \% para efectos de comparación.

Es importante resaltar que en todos los ensayos de esta investigación que involucraban mezclas de Suelo-AMU y que requerían determinar el contenido de agua, se encontró que, para cualquier dosificación de $\mathrm{AMU}$ empleado, en promedio el $24,76 \%$ de éste se evaporaba en el horno a $105^{\circ} \mathrm{C}$, mientras que el otro $75,24 \%$ permanecía junto a los sólidos. Por lo tanto, para el cálculo de las cantidades de material para remoldear los cilindros de compresión inconfinada y del ensayo de consolidación, se tuvieron en cuenta estos valores. Esto se realizó con el fin de obtener unos especímenes con la densidad seca máxima y contenido óptimo de humedad.

Con el ensayo de compresión inconfinada también se determinó el módulo de elasticidad para cada muestra de SueloAMU, de las dos ensayadas para cada porcentaje de AMU se obtuvo una curva esfuerzo-deformación promedio, luego se estimó la pendiente de la parte recta de la curva para la cual el suelo se encuentra en el rango elástico, la cual resulta ser el módulo de elasticidad.

\subsection{Ensayo de permeabilidad mediante la Curva de Taylor}

El coeficiente de permeabilidad $k$ se determinó indirectamente a través de la curva de Taylor, resultado del ensayo de consolidación, basado en la norma INV E - 151.

Para el cálculo de la permeabilidad $k$, solo fue necesario un incremento de carga durante el ensayo de consolida- ción, esto se hace con el fin de determinar el coeficiente de consolidación $C_{v}$ y posteriormente aplicar la siguiente fórmula [1].

$$
k=C_{v} \cdot \gamma_{w} \cdot a_{v} / 1+e_{\mathrm{o}}
$$

Donde,

$C_{\mathrm{v}}$ : Coeficiente de consolidación.

$\gamma_{\mathrm{w}}$ : Peso específico del agua.

$k$ : Coeficiente de permeabilidad.

$a_{\mathrm{v}}$ : Coeficiente de pre consolidación.

$e_{\mathrm{v}}$ : Índice de vacíos antes de la consolidación para un incremento de carga dado.

\section{RESULTADOS}

\subsection{Caracterización del suelo}

El suelo empleado para esta investigación según el "Sistema Unificado de Clasificación de Suelos" resulto ser un limo de alta plasticidad (MH). De acuerdo con la Figura 1 y el ensayo de hidrometría, el suelo contiene un $21,16 \%$ de arcilla, un $61,08 \%$ de limo y un $17,76 \%$ de arena.

Para investigar el efecto del AMU en las propiedades geotécnicas del suelo, se determinaron en primer lugar las propiedades del suelo natural, las cuales se pueden observar en la Tabla 4.

Es natural encontrar este tipo de suelos en el Valle de Lili, debido a que este sector está sobre abanicos aluviales (Sal) y suelos fluvio arcillosos (Sfa), los cuales son sedimentos que han sido transportados y depositados por ríos y quebradas, por ocasionales flujos torrenciales intercalados, haciendo que estos suelos estén conformados por limos arenosos y arcillas (13).

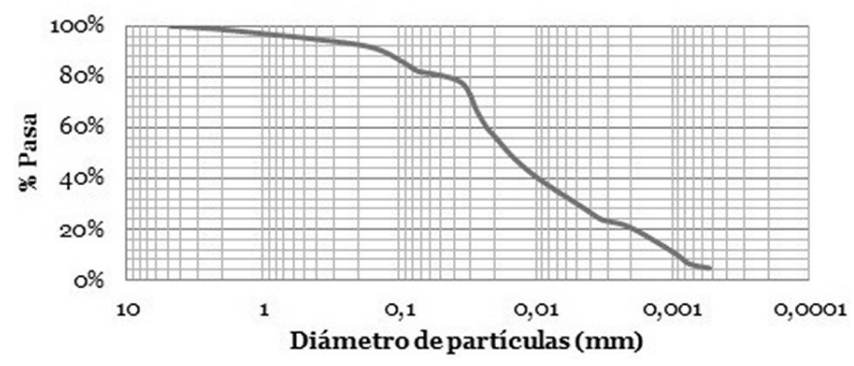

Figura 1. Curva granulométrica.

Tabla 4. Propiedades geotécnicas del suelo sin AMU.

\begin{tabular}{|l|c|c|}
\hline \multicolumn{2}{|c|}{ Resumen del suelo } \\
\hline \multicolumn{2}{|c|}{ Clasificación USCS } & \multicolumn{2}{c|}{ MH } \\
\hline \multirow{2}{*}{ Límites de consistencia } & LL & $52,00 \%$ \\
\cline { 2 - 3 } & LP & $33,48 \%$ \\
\cline { 2 - 3 } & IP & $18,52 \%$ \\
\hline CBR & $4,4 \%$ \\
\hline Densidad seca máxima $\left(\mathrm{kg} / \mathrm{m}^{3}\right)$ & \multicolumn{2}{|c|}{1570} \\
\hline Humedad óptima & $25 \%$ \\
\hline Gravedad específica $G_{s}$ & 2,697 \\
\hline $\begin{array}{l}\text { Resistencia a la compresión inconfinada } q_{u} \\
\text { (Muestra remoldeada en laboratorio) }(\mathrm{kPa})\end{array}$ & \multicolumn{2}{|c|}{1216} \\
\hline Permeabilidad $k(\mathrm{~cm} / \mathrm{seg})$ & $1,5012 \times 10^{-8}$ \\
\hline
\end{tabular}




\subsection{Límites de consistencia o límites de Atterberg}

La clasificación del limo se determinó con la carta de plasticidad de Casagrande como de alta plasticidad (MH). Sin embargo, el índice de plasticidad de 18,52\% no indica que el suelo tenga muy alta plasticidad, esto se debe a que la mayoría de él está compuesto por partículas de tamaño mayor a 0,002 mm, mientras que sólo el $21,16 \%$ son arcillas $(<0,002 \mathrm{~mm})$.

Los límites de consistencia del suelo indican el cambio de un estado físico a otro debido al contenido de agua. Los resultados de los límites líquido y plástico del suelo en función de la cantidad de aceite empleada se muestran en la Figura 2.

En la Figura 2 se observa un ligero incremento del índice de plasticidad con el aumento del contenido de aceite después del $8 \%$ de la dosificación, mientras que para el $4 \%$ y $8 \%$ se mantuvo relativamente constante. En general, se ve claramente que al incrementar el contenido de aceite, tanto los límites líquido y plástico disminuyen. La explicación para este fenómeno es que como las muestras de suelo seco se mezclaron manualmente con el aceite hasta formar una mezcla homogénea y luego se añadió el agua, el aceite fue el primer fluido que entró en contacto con el suelo, por lo tanto, se creó una película alrededor de las partículas del suelo que mitigó la capacidad que tiene el agua para adherirse al suelo. El agua actúa como un agente de unión entre las partículas de arcilla que contribuyen a la plasticidad (8), y se observa que mientras más contenido de aceite se emplee, la cantidad de agua necesaria para que el suelo cambie de un estado a otro es menor, lo cual hace que los límites de Atterberg disminuyan.

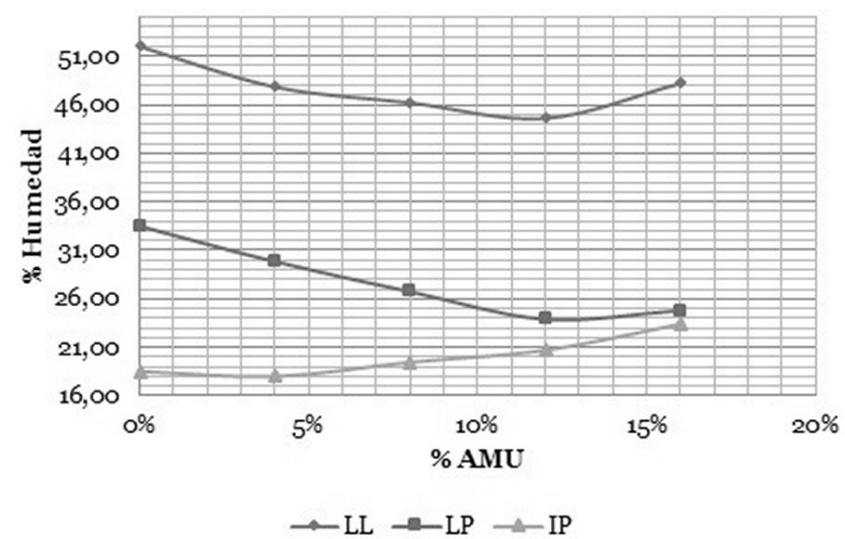

Figura 2. Límites de consistencia en función del AMU.

El AMU aún después de su uso sigue siendo un líquido viscoso y no polar, los suelos arcillosos secos con fluidos no polares no tienen propiedades de plasticidad (3). Además se ha encontrado que si un fluido orgánico se usa en vez de agua, las propiedades del fluido tales como la viscosidad influirán en el límite líquido y los factores físico-químicos harán que la arcilla se comporte más como un material granular, reduciéndose así el límite líquido (3).

\subsection{Densidad seca máxima y contenido óptimo de agua}

Las curvas de compactación en función de la humedad de todos los ensayos, incluido el del suelo natural se resumen en la Figura 3 .

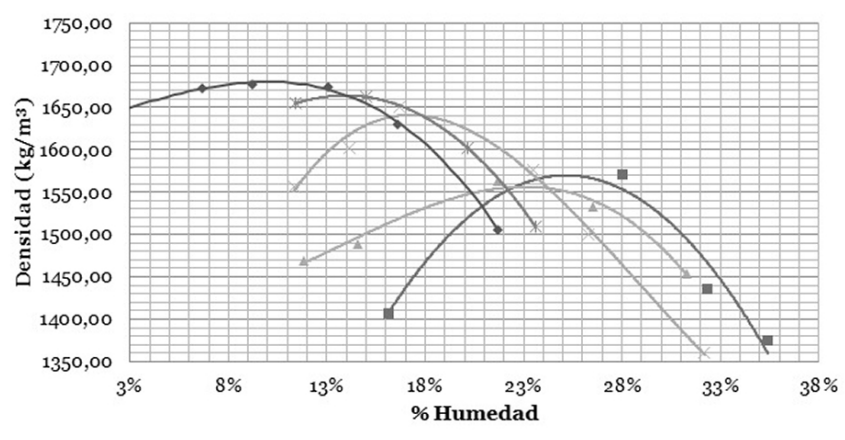

= P.M - $0 \%$ AMU $\triangle$ P.M - $4 \%$ AMU $\times$ P.M - $8 \%$ AMU $\times$ P.M - $12 \%$ AMU • P.M - $16 \%$ AMU

Figura 3. Curvas de compactación (P.M).

De la Figura 3 se nota que al ir incrementando el contenido de AMU, las curvas se van desplazando hacia la izquierda y hacia arriba. La cantidad de agua necesaria para llegar a la densidad seca máxima es menor, en consecuencia, la humedad óptima disminuye con el aumento de aceite y se logra una mayor densidad seca máxima. Como se ve, el decremento de la humedad óptima es significativo para el $4 \%$ de AMU, donde esta se reduce un $8 \%$ con respecto a la del suelo natural, para el $8 \%$ de AMU un $31,2 \%$, para el $12 \%$ de AMU un 44,4\% y finalmente para el $16 \%$ de AMU se reduce un $60 \%$.

En suelos diferentes al limo de esta investigación, otros autores $(3,6,8,10)$ han encontrado resultados similares respecto a la reducción de la humedad óptima en los ensayos de compactación, en estos casos con mezclas de Suelo-Aceite de motor o Suelo-Petróleo crudo. Cuando el suelo se mezcla con el agua, esta cumple la función de lubricar las partículas, formando así una matriz ordenada que permite la compactación del suelo. Al aumentar el contenido de AMU, el agua necesaria para densificar el suelo es menor, es decir que el aceite reemplaza la función del agua y reacomoda las partículas de suelo permitiendo su densificación.

En este orden de ideas, también se observa que la densidad seca máxima tiende a aumentar al incrementar el contenido de AMU. Se observa que para contenidos entre el o \% y $4 \%$ de AMU, la densidad se mantiene relativamente constante, resultado consistente en suelos finos ( $\mathrm{CH}$ y ML) con porcentajes de aceite entre el $4 \%$ y $6 \%$ (10). De la Figura 3 también se nota un aumento de la densidad del 4,5\%, 5,7\% y $7 \%$ con respecto al suelo natural para el $8 \%, 12 \%$ y $16 \%$ de AMU respectivamente.

La composición típica de los aceites usados es una mezcla de lubricantes de diferentes tipos (11), propiedad que hace que el aceite no solo reemplace la función del agua en el ensayo de compactación, sino que lubrique aún más las partículas reacomodándolas y creando una matriz todavía más ordenada del suelo.

Otros investigadores encontraron que la densidad seca máxima tiende a reducirse con el incremento del contenido de aceite. En el caso de los suelos colapsables de Egipto, clasificados como arcillas de baja plasticidad (CL), se atribuye al efecto de la reacción química que ocurre entre el aceite y las partículas de suelo (6), mientras que en ensayos con arena arcillosa (SC) justifican que se debe a que el cambio en la composición del fluido de poro afecta la microestructura del suelo (7). 


\subsection{Resistencia a la compresión inconfinada}

La Figura 4 contiene todas las curvas esfuerzo-deformación obtenidas de los ensayos de compresión inconfinada en función de la cantidad de AMU empleado. Nótese que por cada dosificación ya se han ponderado los resultados de los dos especímenes ensayados, incluso para el suelo natural.

Los resultados de resistencia $q_{\mathrm{u}}$, su deformación unitaria correspondiente $\varepsilon$ y los módulos de elasticidad $E$, se encuentran resumidos en la Tabla 5. Los valores de resistencia clasifican al suelo natural y a las mezclas de Suelo-AMU con una consistencia dura según el INVIAS (16). Sin embargo, la consistencia de todas las muestras se debe a que cada una se compacto entre el $94 \%$ al $100 \%$ del Proctor Modificado.

Los valores de resistencia a la compresión inconfinada y deformación unitaria en función de la cantidad de AMU empleado se graficaron en la Figura 5.

De la Figura 5, en general se observa que hay un decremento significativo de la resistencia únicamente después del $8 \%$ de aceite en comparación con la del suelo natural, donde cae un $16 \%$ y un $29 \%$ para contenidos de AMU del $12 \%$ y $16 \%$ respectivamente. En los porcentajes del $4 \%$ y $8 \%$ de AMU, la resistencia se mantiene en el $94 \%$ de la muestra patrón. En conse-

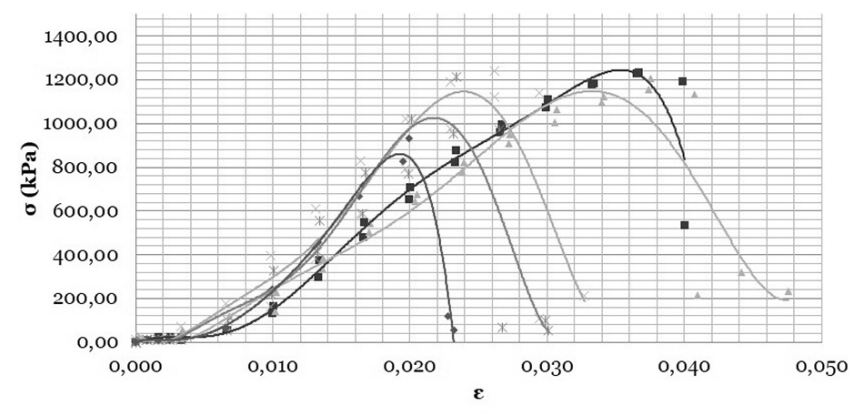

- C.I - o\% AMU $\triangle$ C.I - $4 \%$ AMU $\times$ C.I - $8 \%$ AMU $*$ C.I - $12 \% \mathrm{AMU}+$ C.I - $16 \% \mathrm{AMU}$

Figura 4. Curvas esfuerzo-deformación en función del AMU.

Tabla 5. Resultados pruebas de compresión inconfinada.

\begin{tabular}{|c|c|c|c|}
\hline \%AMU & $\mathbf{q}_{\mathbf{u}}(\mathbf{k P a})$ & $\varepsilon$ & $\mathbf{E}(\mathbf{M P a})$ \\
\hline $0 \%$ & 1216 & 0,037 & 47 \\
\hline $4 \%$ & 1138 & 0,034 & 40 \\
\hline $8 \%$ & 1138 & 0,025 & 54 \\
\hline $12 \%$ & 1020 & 0,022 & 61 \\
\hline $16 \%$ & 863 & 0,020 & 64 \\
\hline
\end{tabular}

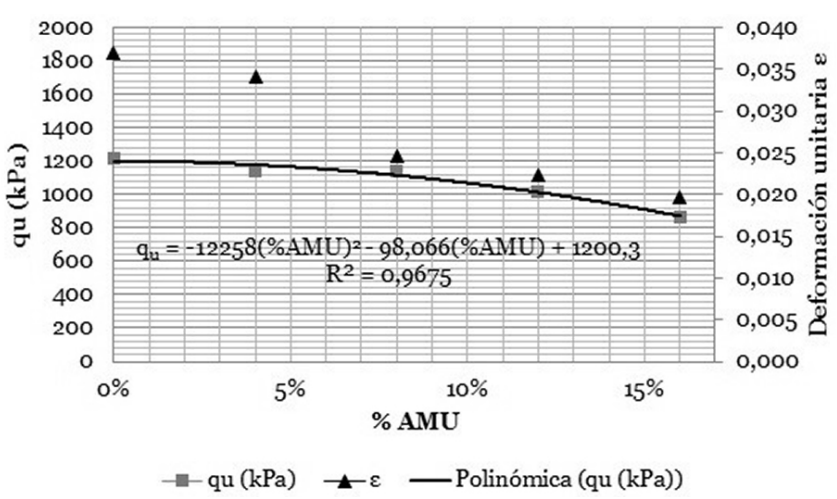

Figura 5. Resistencia $q_{\mathrm{u}} \mathrm{y}$ deformación $\varepsilon$ en función del AMU. cuencia se puede inferir que para contenidos de aceite del o\% al $8 \%$, la resistencia se mantendrá relativamente constante.

La línea de tendencia trazada en la Figura 5, comprueba numéricamente el análisis anterior referente a la resistencia a la compresión inconfinada, y se describe por la siguiente ecuación polinómica [2].

$$
\text { [2] } \quad q_{u}=-12258(\% A M U)^{2}-98,066(\% A M U)+1200,3
$$

Donde,

$q_{\mathrm{u}}$ : Resistencia a la compresión inconfinada, expresada en kPa. $\% A M U$ : Aceite de Motor Usado, expresado en tanto por ciento.

A medida que se usa más cantidad de aceite en el suelo, la película lubricante alrededor de las partículas facilita su deslizamiento, causando que la deformación unitaria que permite el suelo antes de fallar sea menor.

Respecto a la resistencia al corte en arcillas CL, muestra que al incrementar el contenido de aceite hay una extrema reducción de la cohesión, mientras que suelos SP y SM presentan una baja cohesión, que puede ser resultado de la viscosidad y la cohesión inherente (3). El decremento del esfuerzo y deformación de suelos finos puede ser causado por la naturaleza viscosa del fluido de poro empleado. Un aumento en la viscosidad del fluido de poro, cambia las propiedades de contacto y las interacciones fisicoquímicas, mostrando con ello un ablandamiento de la muestra (9).

\subsection{Coeficiente de permeabilidad}

Con los datos obtenidos de todas las muestras se realizaron los cálculos mediante la fórmula [1] del capítulo de metodología. Los valores de este ensayo para cada dosificación de AMU, se resumen en la Tabla 6.

Tabla 6. Resultados de ensayo de permeabilidad - Curva de Taylor.

\begin{tabular}{|c|c|c|c|}
\hline \%AMU & $\mathbf{G}_{\mathbf{s}}$ & $\mathbf{e}_{\mathbf{o}}$ & $\mathbf{k}(\mathbf{c m} / \mathbf{s e g})$ \\
\hline $0 \%$ & 2,69 & 0,77 & $1,501 \times 10^{-8}$ \\
\hline $4 \%$ & 2,50 & 0,58 & $3,295 \times 10^{-8}$ \\
\hline $8 \%$ & 2,33 & 0,48 & $3,239 \times 10^{-8}$ \\
\hline $12 \%$ & 2,18 & 0,42 & $2,380 \times 10^{-8}$ \\
\hline $16 \%$ & 2,05 & 0,24 & $0,605 \times 10^{-8}$ \\
\hline
\end{tabular}

Nótese que la gravedad específica del suelo natural fue calculada como se describió en el numeral 3.3, pero para mezclas de Suelo-AMU se aplicó la fórmula [3] encontrada en (10).

[3]

$$
G_{s}=1 /\left(\left(P_{1} / 100 \cdot G_{1}\right)+\left(\left(P_{2} / 100 \cdot G_{2}\right)\right)\right.
$$

Donde,

$G_{\mathrm{s}}$ : Gravedad específica de la mezcla de suelo aceite.

$P_{1}$ : Masa en porcentaje de las partículas de suelo.

$P_{2}$ : Masa en porcentaje del aceite.

$G_{1}$ : Gravedad específica de las partículas de suelo $(2,697)$.

$G_{2}$ : Gravedad específica del aceite $(0,9142)$.

Dado que el suelo empleado en esta investigación resultó ser un $\mathrm{MH}$ con más del $80 \%$ de partículas con tamaño inferior a 
$75 \mu \mathrm{m}$, evaluar la influencia del aceite en la permeabilidad de manera directa resulta demasiado extenso en términos de tiempo.

El coeficiente de permeabilidad calculado para el suelo natural fue de $1,50119 \times 10^{-8}$, el cual es un valor típico para un suelo fino que lo clasifica como impermeable (17).

Claramente se ve que no hay una influencia significativa del contenido de AMU en el coeficiente de permeabilidad. Esto puede deberse tanto al alto porcentaje de partículas finas, como a que todas las muestras se compactaron a más del $94 \%$ de la densidad seca máxima, lo cual hace que se presente un coeficiente muy bajo sin importar la cantidad de aceite empleado.

Sin embargo, a pesar de que la permeabilidad en general no mostró una variación representativa, si se presentó una reducción considerable del volumen de vacíos al incrementar el contenido de AMU, éste se redujo de manera proporcional como se indica en la Figura 6.

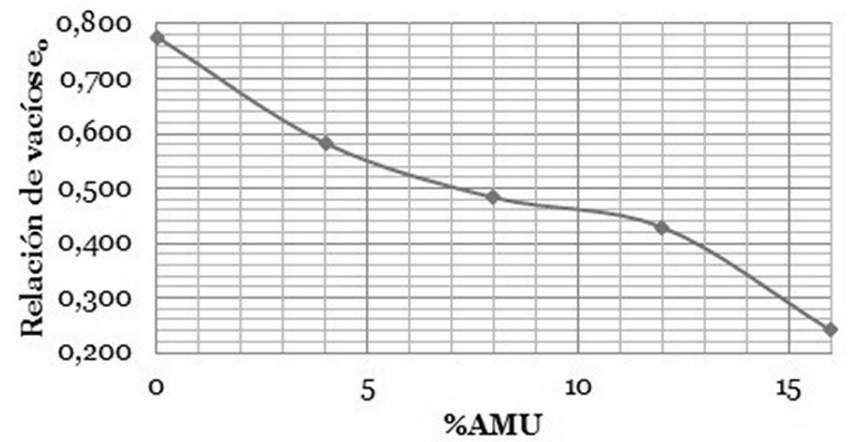

Figura 6. Relación de vacíos en función del AMU.

Como se ve en la Figura 6, al incrementar la dosificación de aceite, el volumen de vacíos disminuye. Por ejemplo, para el $4 \%$ de AMU el volumen de vacíos se reduce un $25 \%$ con respecto al del suelo natural, para el $8 \%$ de AMU un $38 \%$, para el $12 \%$ de AMU un $45 \%$ y finalmente para el $16 \%$ de AMU se reduce un $69 \%$. Estos resultados concuerdan con los obtenidos en algunas investigaciones previas: un incremento en el contenido de aceite en suelos residuales $\mathrm{MH}$, reducirá los espacios entre partículas que permiten la filtración de agua (8). En el estudio de los efectos de petróleo crudo en suelos CL, SM y SP, se obtuvo una correlación directa entre la permeabilidad y el tamaño de partículas y una correlación inversa entre la permeabilidad y el contenido de aceite $(3,4)$, pero la reducción del coeficiente de permeabilidad con el incremento de aceite no fue significativo, incluso para el $16 \%$; también se señaló que la reducción de la permeabilidad es mayor en suelos como SM o SP, lo que indica el efecto del aceite en la permeabilidad decrece con el incremento de la porosidad. En un suelo con un alto porcentaje de partículas de arcilla, no disminuye significativamente la permeabilidad con el contenido de aceite, sin embargo, el principal factor que influye en la permeabilidad de un suelo es el cambio de la estructura del mismo por el contenido de agua (10).

$\mathrm{Al}$ parecer, aunque el AMU reduce el bajo volumen de vacíos de un suelo $\mathrm{MH}$, no se presenta un cambio significativo del coeficiente de permeabilidad $k$. Los limos al estar compuestos por un alto contenido de partículas finas presentan un coefi- ciente de permeabilidad muy bajo, además es necesario tener en cuenta que el cálculo de la permeabilidad depende de varios factores.

La gran ventaja de este tipo de estabilización para aplicaciones viales es que se logra una capa impermeable y resistente, que no se satura y protege de la saturación por lluvia la capa superior de la subrasante, lo cual la hace estable dándole mejor soporte a la estructura de pavimento.

\section{CONCLUSIONES}

En este estudio se seleccionó un suelo típico de subrasante en la construcción de vías de Colombia, particularmente en la ciudad de Santiago de Cali. El suelo fue clasificado como un limo de alta plasticidad ( $\mathrm{MH}$ ) según el Sistema Unificado de Clasificación de Suelos (USCS).

Se evaluó de forma exploratoria el efecto de un Aceite de Motor Usado (AMU) en el suelo MH mediante ensayos geotécnicos preliminares. Con los resultados obtenidos, se incentiva a que futuras investigaciones enfoquen sus esfuerzos en otros aspectos geotécnicos, como las propiedades mecánicas, efecto a largo plazo de otros tipos de suelos en presencia de AMU $\mathrm{y}$ aspectos ambientales.

Para el suelo investigado, los límites de consistencia líquido y plástico tienden a disminuir con el incremento de AMU, lo cual se debe a que las partículas de suelo son cubiertas con una película viscosa y no miscible con el agua, que disminuye su capacidad para adherirse al suelo y darle fluidez.

Se presentó un ligero aumento de la densidad seca máxima (hasta un $7 \%$ ) para dosificaciones mayores al $4 \%$ de AMU, mientras que para contenidos entre el o\% y $4 \%$ se mantuvo constante. Se obtuvo una disminución significativa de la humedad óptima al incrementar el contenido de aceite, en donde se redujo hasta un $60 \%$ para el $16 \%$ de AMU. Esto se atribuye a que el aceite de motor reemplaza la cantidad de agua necesaria para densificar el suelo y lubrica aún más las partículas del mismo, permitiendo su reacomodo a bajos contenidos de agua.

Se observó que la consistencia dura de todas las muestras remoldeadas se debe al grado de densificación de las mismas (94-100\% del P.M). Para contenidos de AMU desde el o\% hasta el $8 \%$, la resistencia a la compresión inconfinada $\left(q_{\mathrm{u}}\right)$ se mantuvo relativamente constante, después de lo cual en comparación con el suelo natural cae un $16 \%$ y $29 \%$ para el $12 \%$ y $16 \%$ de AMU, respectivamente.

La permeabilidad del suelo no se afectó significativamente con el contenido de aceite debido a que el limo de alta plasticidad de los ensayos estuvo compuesto por más del $80 \%$ de partículas finas, además todas las muestras fueron compactadas a la densidad seca máxima, reduciendo al máximo el coeficiente de permeabilidad. Sin embargo, el volumen de vacíos disminuyó considerablemente con el aumento del aceite, lo cual hace que en suelos más porosos sea posible disminuir el coeficiente de permeabilidad.

Los resultados de esta investigación exploratoria muestran que se justifica realizar una mayor investigación sobre este tema, dado que puede existir un uso ingenieril de este tipo de estabilización. 


\section{REFERENCIAS}

(1) Rico Rodríguez, A., \& Del Castillo, H. (2005). Planteamiento teórico del problema del flujo de agua en suelos. En La ingeniería de suelos en las vías terrestres: carreteras, ferrocarríles y aeropistas (pág. 387). México D.F: Limusa S.

(2) Aiban, S. A. (1998). The effect of temperature on the engineering properties of oil-contaminated sands. Environment international, 24(1-2): 153-161. https://doi.org/10.1016/So160-4120(97)00131-1

(3) Khamehchiyan, M., Charkhabi, A. H., \& Tajik, M. (2007). Effects of crude oil contamination on geotechnical properties of clayey and sandy soils. Engineering Geology, 89(3-4): 220-229. https://doi.org/10.1016/j.enggeo.2006.10.009

(4) Khamehchiyan, M., Charkhabi, A. H., \& Tajik, M. (2006). The effects of crude oil contamination on geotechnical properties of Bushehr coastal soils in Iran. Geol. Soc. London, 214, 1-6.

(5) Nazir, A. K. (2011). Effect of motor oil contamination on geotechnical properties of over consolidated clay. Alexandria Engineering Journal, 50(4): 331-335. https://doi.org/10.1016/j.aej.2011.05.002

(6) Nazir, A. K. (2012). Stabilization of Collapsible Soil with Engine Oil. International Review of Civil Engineering, 3(6): 487-492.

(7) Ojuri, O. O., \& Ogundipe, O. O. (2012). Modelling used engine oil impact on the compaction and strength characteristics of a lateritic soil. EJGE, 17: 3491-3501.

(8) Rahman, Z. A., Hamzah, U., Taha, M. R., Ithnain, N. S., \& Ahmad, N. (2010). Influence of oil contamination on geothecnical properties of basaltic residual soil. American journal of applied sciences, 7(7): 954-961. https://doi.org/10.3844/ ajassp.2010.954.961

(9) Ratnaweera, P., \& Meegoda, J. N. (2006). Shear strength and stress-strain behavior of contaminated soils. Geotechnical Testing Journal, 29(2): 133-140. https://doi.org/10.1520/GTJ12686

(10) Silvestry, V., Mikhail, N., \& Soulié, M. (1997). Permeability response of oil-contaminated compacted clays. In Testing soil mixed with waste or recycled materials, 62-75. ASTM International. https://doi.org/10.1520/STP15643S

(11) Nuñez, M.E. (2001). Aceite usado generado por motores en la ciudad de Cali. Alternativas de uso. Revista de la Bolsa de Residuos y Subproductos Industriales, 3: 4-11.

(12) UPME, Unidad de planeación minero-energética. (2001). Transformación de los aceites usados para su utilización como energéticos en procesos de combustión. Bogotá D.C.

(13) Instituto Colombiano de Geología y Minería, Ingeominas. (2005). Estudio de microzonificación sísmica. Informe 4 Investigaciones y Zonificación Geotécnica de la Ciudad de Santiago de Cali. Bogotá.

(14) ASTM D1298. (2012). Standard test method for density, relative density, or API gravity of crude petroleum and liquid petroleum products by hydrometer method. Annual Book of Standards. https://doi.org/10.1520/D1298-12B

(15) INVIAS. (2013). Normas de Ensayo de materiales para carreteras.

(16) INVIAS. (2007). INV E - 152. Compresión inconfinada en muestras de suelos.

(17) Angelone, S., Garibay, M.T., \& Casaux, M.C. (2006). Permeabilidad de suelos. Recuperado de http://www.fceia.unr.edu. ar/geologiaygeotecnia/Permeabilidad\%20en\%20Suelos.pdf 